\title{
Three-dimensional and Higher-order Imaging with Tomographic SAR: Techniques, Applications, Issues
}

\author{
Andreas Reigber ${ }^{1}$, Fabrizio Lombardini ${ }^{2}$, Federico Viviani ${ }^{2}$, Matteo Nannini ${ }^{1}$, Antonio Martinez del Hoyo ${ }^{1}$ \\ ${ }^{1}$ German Aerospace Centre (DLR) Microwaves and Radar Institute, Oberpfaffenhofen, Germany \\ ${ }^{2}$ University of Pisa, Department of Information Engineering, Pisa, Italy
}

\begin{abstract}
SAR tomography is a remote sensing technique, extending SAR interferometry, that allows three-dimensional imaging of multiple height-distributed point-like scatterers or volumetric targets. It allows, for example, to solve layover urban scatterers or to analyze the vertical structure of vegetation layers or other targets with significant penetration of the sensor's radiation (dry soil, ice layers, etc.). The ability to monitor the 3-D inner structure of volumetric targets and to extract information about the nature and location of ongoing scattering processes promises a break-through in key environmental problems. Indeed, structural parameters of volume scatterers in the biosphere and cryosphere, such as vegetation height and moisture content, forest vertical structure and biomass, or snow / ice depth and layering are critical inputs for ecological process modeling and enable effective monitoring of ecosystem change. The 3-D tomographic principle has been also extended to higher dimension in the more recent differential tomography methodology, producing 4-D (3-D + time) images bridging the gap between differential interferometry and SAR tomography.

Due to their unique capabilities and potential, tomographic SAR techniques have gained significant attention during the last years and become established methods for analysis of all kinds of three-dimensional back-scattering scenarios. In particular, for future space-borne SAR sensors operating in longer wavelength, like BIOMASS, SAOCOM-CS or Tandem- $\mathrm{L}$, it is planned to employ 3-D tomographic imaging modes on an operational basis. This paper aims to review the different state-of-the-art signal processing methods for threedimensional SAR imaging, which have been developed during the last decade, and for four-dimensional differential tomography. The requirements, advantages and disadvantages of different techniques are assessed and compared and open issues to be addressed in future developments are pointed out.
\end{abstract}

\section{TOMOGRAPHIC TECHNIQUES FOR IMAGING OF VERTICAL STRUCTURES}

The most common geometrical setup for tomographic SAR imaging is multi-baseline imaging. As illustrated in Fig.1, here a scene is repeatedly imaged from slightly different viewing positions, together spanning a synthetic aperture $L$ in the direction perpendicular to range and azimuth. With simple geometric considerations, it can be demonstrated that the SAR multi-baseline (complex valued) data vector and the complex reflectivity of the

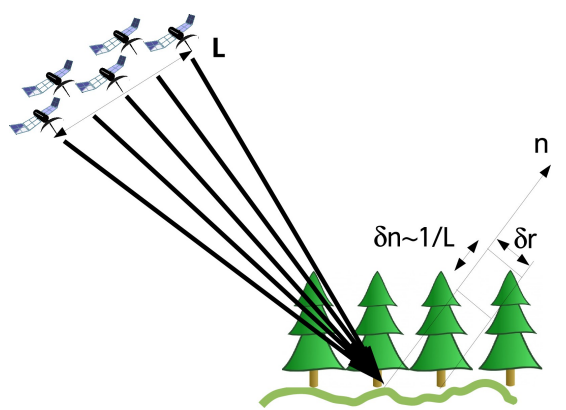

Fig. 1. Multi-baseline tomographic imaging geometry. Resolution capability in the $n$-direction, perpendicular to range, is inversely proportional to the total baseline span $L$.

imaged target along the vertical axis constitute a Fourier pair, formally leading to a Rayleigh resolution along the $n$-axis proportional to $1 / L$. Since the first experimental trials apart from laboratory conditions in the late 90'ies [1], [2], [3], various techniques for tomographic focusing having been developed, as discussed in the following.

\section{A. Spatial spectral analysis}

The tomographic synthetic aperture directly justifies multi-baseline array processing, which can be considered a coherent SAR data combination, in particular based on spectral estimation. Employed techniques started with classical Fourier beamforming [3] and some improvements with regularization [4]. Fourier focusing is closely related to least-square estimation of signals coming from a certain direction of arrival (DOA), which does not require any particular assumptions on the vertical scatterer distribution. However, Fourier focusing is known to generate significant ambiguities under irregular sampling conditions and possesses only a limited height resolution capability. Therefore, soon modern spectral estimators have been used in SAR tomography, especially Capon [5], [6], [7], being able to adaptively reject side-lobes from directions different to the selected DOA and to overcome the vertical Rayleigh diffraction resolution limit. Also other powerful super- 
resolution model-based alternatives have been applied or adapted to SAR tomography, like the multiple signal classification (MUSIC) [5], [8], [7] and the weighted subspace fitting (WSF) [9], both being model-based in the sense that they consider the signal as a superposition of several point-like sources. As such, the model order is important in these methods [5]; order detection algorithms have so been injected in the tomographic literature, like e.g. Information Theoretic Criteria (ITC) methods [10]. It has to be noted, that in contrast to Fourier focusing, the current adaptive estimation techniques are not directly able to preserve radiometric calibration, a central requirement in many (natural) applications scenarios. For urban applications, the well tested Capon and MUSIC methods have been also extended to single-look processing [8].

\section{B. Coherence tomography}

An alternative to classical tomographic approaches can be addressed in terms of single interferograms, i.e. exploring the first order off-diagonal structure of the observation covariance matrix. This leads to 3-D imaging approaches that can be framed in a correlative imaging [11] area rather than in the array beamforming processing field. Such approaches are especially beneficial in case of tandem imaging constellations, i.e. when having high-coherent single-pass InSAR pairs, but large temporal decorrelation between the passes (TanDEM-X, SAOCOM-CS, TandemL). A special case, powerful in case of a low number (1-3) of interferograms, is the strongly model-based (polarimetric) coherence tomography (PCT) [12], [13]: with knowledge of ground height and volume thickness, the vertical reflectivity is approximated by a normalized Legendre polynomial series. The individual interferograms are used to estimate the coefficients of the individual components.

\section{Compressed sensing}

Compressed sensing (CS) is a new and generalized approach to sensing problems, which has successfully been used also in the field of SAR tomography [14], although computationally burdensome. It is assuming the vertical scatterer distribution to be sparse and derives the parameters of these sparse components through an optimisation process. Although originally adapted to (urban) sparse point-like scatterer distributions [15], [14], newer developments extend the compressed sensing inversion concept also for distributed scatterer scenarios by instead reconstructing in a sparse wavelet basis [16]. The wavelet-based compressive sensing (WCS) method is basically a covariance fitting approach, that exploits the low dimensionality of the cross-range power distribution for a specific polarimetric channel in the wavelet domain. Depending on the complexity of the scene, CS can be powerful especially when only a rather low number of acquisitions is available.

\section{Sum-of-Kronecker-products decomposition}

An alternative approach to direct array spectral estimation or CS inversion for 3-D image reconstruction is, for the forest scenario case, to use baseline (angular) and polarization diversity to filter ground and canopy components by exploiting their uncorrelated polarimetric responses. Under this assumption, the covariance matrix of the data can be modeled as a sum of two Kronecker products, composed of a polarimetric and a structural component for ground and canopy, respectively. As a result, after this blind filtering, standard tomographic techniques can be subsequently applied on the structural components separately [17]. Also the SKP technique has shown a great potential when only few baselines are available. Moreover it can successfully be coupled with compressed sensing approaches [18].

A comparison of a few of the above mentioned methods for 3-D tomography is shown in Fig. 2, for a same airborne dataset and a volumetric forest scenario. Two other examples of tomographic results are reported in Fig. 3 top, for spaceborne and airborne data and a layover urban/bridge infrastrucure area.

\section{DIFFERENTIAL-TOMOGRAPHIC TECHNIQUES FOR VERTICAL NON-STATIONARY STRUCTURES}

When operating the mature spaceborne multipass interferometric SAR sensing, a typical goal is monitoring slow deformation motions of high interest, e.g. (urban) subsidences or structural deformations [19], [20]. However, although SAR tomography can separate multiple scatterers, it has no measuring sensitivity to their deformation motions. In addition to this, the tomographic imaging process can be affected by them, producing height misplacement and blurring effects. On the other hand, differential interferometry (D-InSAR) techniques sense deformations, but degrade or cannot operate in multiple scattering scenarios.

For these reasons, a new coherent data combination mode termed differential SAR tomography (Diff-Tomo) has been originated [21], synergically integrating the differential interferometry and the tomographic concepts to allow "opening" the SAR pixel in complex non-stationary scenes, degarbling its information content. Diff-Tomo is based on two-dimensional space-time spectral analysis, fully exploiting the multibaseline-multipass data. It allows the joint resolution of multiple heights and deformation velocities of the scatterers mapped in a SAR pixel, i.e. 4-D (3-D + time, multidimensional) SAR imaging. To handle frequent heavy ambiguities from the very sparse baseline-time sampling, 2D superresolution methos have been developed, Capon [21], MUSIC, also at singlelook [22], and CS [23]. Typical Diff-Tomo application is the reconstruction and monitoring of urban/infrastructure deformating scenarios in presence of layover [24], [25], [23], [22]. 

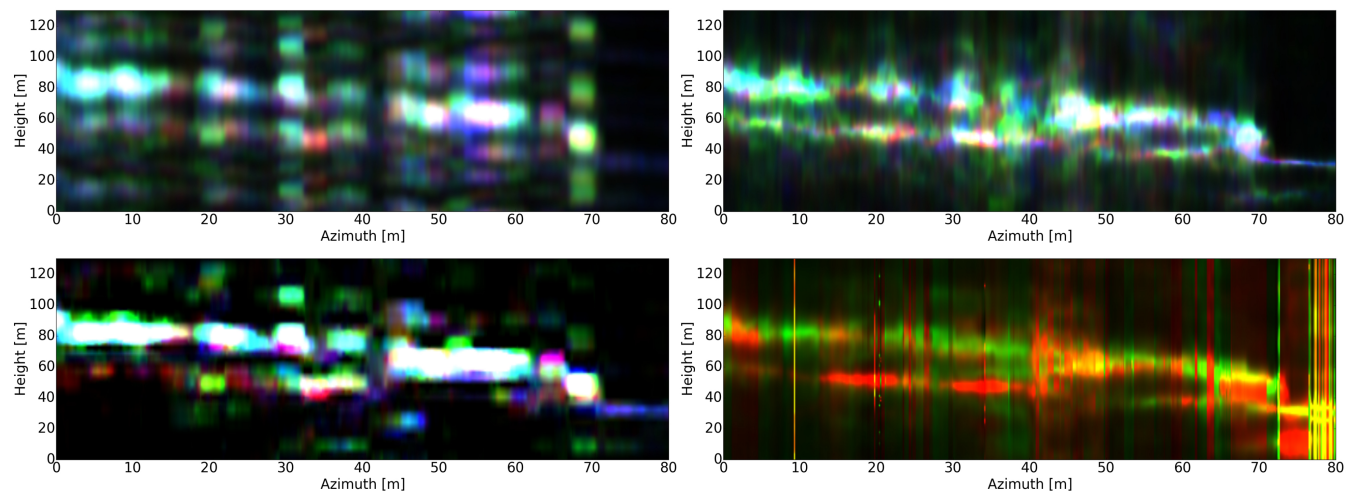

Fig. 2. 3-D tomographic height/azimuth slices derived from 10 irregularly spaced tracks with up to 55m baseline, acquired by DLR F-SAR sensor at L-band. Colours correspond to the polarimetric Pauli channels. Shown is a forested area with decreasing ground topography from left to right with a small bare surface area at the right image border. Top left: Fourier, showing strong ambiguities due to irregular track sampling. Top right: Capon. Bottom left: Wavelet-based compressive sensing. Bottom right: SKP followed by Capon on ground (red) and canopy (green) components model-mismatch leads to strong artefacts on surface areas.
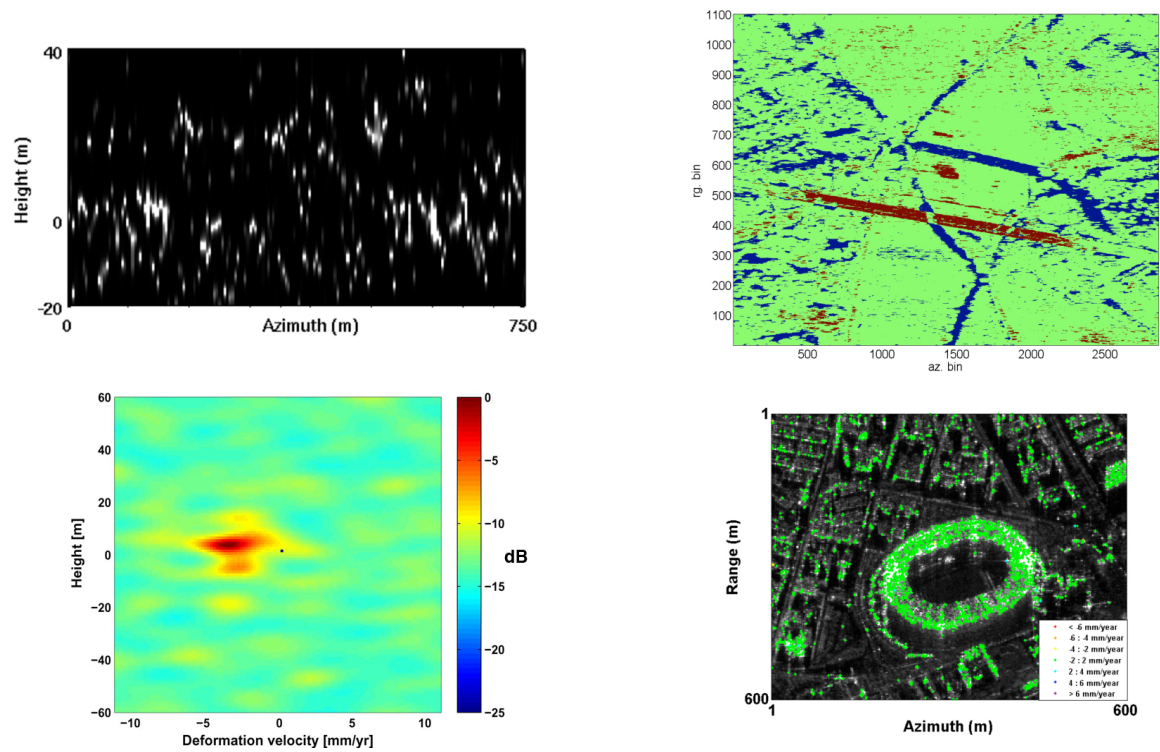

Fig. 3. 3-D tomographic and 4-D differential tomographic results over urban/infrastructure scenes. Top left: 3-D height/azimuth slice from 40 tracks with $1500 \mathrm{~m}(\max )$ baseline, ERS-1/2 C-band satellite, dense building area, single-look MUSIC (constant model order), ground and roof layers apparent. Top right: 3-D detected order map from three-antenna interferometer with $0.8 \mathrm{~m}$ max baseline, FGAN AER-II X-band sensor, brown: double scatterer, green: single scatterer, blue: noise only, bridge over valley scene, eigen-based ITC (EDC) model order selection. Bottom left: 4-D height/deformation velocity frame from 30 tracks with 1065m max baseline, 6yr time span, ERS-1/2 C-band, building double scatterer SAR cell, Capon. Bottom right: 4-D deformation velocity map from 28 tracks with $1300 \mathrm{~m}$ max baseline, 1yr time span (ack ASI), COSMO-SkyMed X-band satellites, differences wrt low-res D-InSAR reference, double scatterer dominant component, urban stadium area, single-look Capon/order detector.

While for the case of multiple sparse point-like scatterers in layover affected by (possibly different) uniform deformation motions the space-time spectrum to be identified by 4-D Diff-Tomo processing is discrete, the differential tomographic sensing concept is not intrinsically limited to such scenarios. More generally, Diff-Tomo can identify scattering component distributions in the domain of spatial (height) and temporal frequency of harmonics in which a signal from a scatterer with temporal decorrelation [21], [22] or non-uniform motion [24], [22] can be decomposed, also avoiding their misinterpretation in tomographic processing [22], [26], and potentially allowing new mapping and monitoring possibilities. An example of 4-D differential tomography output and a validation of 4-D extracted information are reported in Fig. 3 bottom, both for spaceborne data, and a layover urban scene.

\section{ApPliCATIONS \& OUTLOOK}

As already exemplified in Figs. 2-3, typical applications of (3-D) tomographic SAR methods have been related so far both to layover infrastructure/urban scenes and volumetric forest areas, exploiting mainly space-borne and airborne data respectively; recently also snow/ice 
experimental application has started [27]. Extended (4-D) differential-tomographic spaceborne techniques have been recently applied to solve urban layover even at the singlebuilding level with TerraSAR-X and COSMO-SkyMed data.

For many years space-borne implementation of SAR tomography was typically limited by temporal decorrelation induced by large repeat-pass cycles and sensor's short wavelengths, especially for the vegetated areas. However, for the new generation of space-borne SAR missions, tomographic modes are getting more and more feasible or even an experimental or operational mode also for forest applications. In ESA's BIOMASS mission project for global biomass mapping, SAR tomography is crucial as forest height and structure estimates are the key to assess tropical ecosystems. The potential of tomographic SAR techniques is fully developed in the frame of DLR's Tandem-L mission proposal, enabling systematic and global monitoring of dynamic structure processes in Earth's bio- and cryosphere.

Among emerging tomographic research topics, the possibility has been shown to exploit 4-D imaging to obtain 3-D multipass forest tomographic imaging exploiting all the baselines combinations robust to (long-term) temporal decorrelation [22], [26]. Another new developing application concerns the potential of Diff-Tomo to extract dynamic information in layered media, in particular separating in the height dimension different temporal decorrelation mechanisms [22] that are mixed (undiscriminated) in the classical (ensemble) coherence analysis, allowing new important phenomenological investigations of forest scenes.

\section{REFERENCES}

[1] J. Homer, I. Longstaff, and G. Callaghan, "High resolution 3D SAR via multi-baseline interferometry," Proceedings of IGARSS'96, Lincoln, USA, pp. 796-798, 1996.

[2] L. Roessing and J. Ender, "Advanced sar interferometry techniques with aer-ii," Proceeding of International Radar Symposium, p. 1261-1269, 1998.

[3] A. Reigber and A. Moreira, "First demonstration of airborne SAR tomography using multibaseline L-band data," IEEE Transactions on Geoscience and Remote Sensing, vol. 38, no. 5, pp. 2142-2152, 2000.

[4] G. Fornaro, F. Serafino, and F. Soldovier, "Three-dimensional focusing with multipass sar data," IEEE Transactions on Geoscience and Remote Sensing, vol. 41, no. 3, pp. 507-517, 2003.

[5] F. Gini, F. Lombardini, and M. Montanari, "Layover solution in multibaseline SAR interferometry," IEEE Transactions on Aerospace and Electronic Systems, vol. 38, pp. 1344-1356, 2002.

[6] F. Lombardini and A. Reigber, "Adaptive spectral estimation for multibaseline SAR tomography with airborne L-band data," Proceedings of International Geoscience and Remote Sensing Symposium, vol. 3, pp. 2014-2016, July 2003.

[7] M. Nannini, R. Scheiber, R. Horn, and A. Moreira, "First 3$\mathrm{D}$ reconstructions of targets hidden beneath foliage by means of polarimetric sar tomography," Geoscience and Remote Sensing Letters, IEEE, vol. 9, no. 1, pp. 60-64, 2012.

[8] F. Lombardini, F. Cai, and D. Pasculli, "Spaceborne 3-d sar tomography for analyzing garbled urban scenarios: Single-look superresolution advances and experiments," IEEE Journal of Selected Topics in Applied Earth Observations and Remote Sensing, vol. 6, no. 2, pp. 960-968, April 2013.
[9] Y. Huang, A. Ferro-Famil, and A. Reigber, "Under-foliage object imaging using SAR tomography and polarimetric spectral estimators," IEEE Transactions on Geoscience and Remote Sensing, vol. 50, no. 6, pp. 2213-2225, June 2012.

[10] F. Lombardini, L. Rossing, J. Ender, and F. Cai, "Interferometric model order selection: Validation of itc methods with airborne three-antenna sar data," in IEEE International Conference on Geoscience and Remote Sensing Symposium, 2006. IGARSS 2006. IEEE, 2006, pp. 2565-2568.

[11] R. Treuhaft, B. Chapman, J. dos Santos, L. Dutra, F. Goncalves, C. da Costa Freitas, J. Mura, P. de Graca, and J. Drake, "Tropicalforest density profiles from multibaseline interferometric sar," Proceedings of International Geoscience and Remote Sensing Symposium, pp. 2205-2207, July 2006

[12] S. Cloude, "Dual baseline coherence tomography," IEEE Geoscience and Remote Sensing Letters, vol. 4, no. 1, pp. 127-131, January 2007.

[13] M. Pardini, A. Torano-Caicoya, F. Kugler, S. K. Lee, I. Hajnsek, and K. Papathanassiou, "On the estimation of forest vertical structure from multibaseline polarimetric SAR data," Proceedings of International Geoscience and Remote Sensing Symposium, pp. 3443-3446, July 2012.

[14] A. Budillon, A. Evangelista, and G. Schirinzi, "Three-dimensional SAR focusing from multipass signals using compressive sampling," IEEE Trans. Geosci. Remote Sens., vol. 49, no. 1, pp. 488-499, Jan. 2011.

[15] X. Zhu and R. Bamler, "Tomographic SAR inversion by L1-norm regularization - The compressive sensing approach," IEEE Trans. Geosci. Remote Sens., vol. 48, pp. 3839-3846, Oct. 2010.

[16] E. Aguilera, M. Nannini, and A. Reigber, "Wavelet-based compressed sensing for sar tomography of forested areas," IEEE Transactions on Geoscience and Remote Sensing, vol. 51, no. 12, Dec 2013

[17] S. Tebaldini, "Algebraic synthesis of forest scenarios from multibaseline PolInSAR data," IEEE Trans. Geosci. Remote Sens. vol. 47, pp. 4132-4142, Dec. 2009.

[18] E. Aguilera, M. Nannini, and A. Reigber, "A data-adaptive compressed sensing approach to polarimetric SAR tomography of forested areas," IEEE Geosci. Remote Sens. Lett., vol. 10, no. 3, pp. 543-547, May 2013.

[19] P. A. Rosen et al., "Synthetic aperture radar interferometry," Proceedings of the IEEE, vol. 88, no. 3, pp. 333-382, 2000.

[20] A. Ferretti, C. Prati, and F. Rocca, "Nonlinear subsidence rate estimation using permanent scatterers in differential sar interferometry," IEEE transactions on Geoscience and Remote Sensing, vol. 38, no. 5, pp. 2202-2212, 2000.

[21] F. Lombardini, "Differential tomography: a new framework for sar interferometry," IEEE Transactions on Geoscience and Remote Sensing, vol. 43, no. 1, pp. 37-44, Jan 2005.

[22] F. Lombardini, F. Cai, F. Viviani, and D. Pasculli, "Multidimensional sar tomography for complex non-stationary scenes: Cosmoskymed urban and p-band forest results," Proceedings of International Geoscience and Remote Sensing Symposium, pp. 5206-5209, July 2012.

[23] X. X. Zhu and R. Bamler, "Demonstration of super-resolution for tomographic sar imaging in urban environment," IEEE Transactions on Geoscience and Remote Sensing, vol. 50, no. 8, pp. 3150-3157, Aug 2012

[24] G. Fornaro, D. Reale, and F. Serafino, "Four-dimensional sa imaging for height estimation and monitoring of single and double scatterers," IEEE Transactions on Geoscience and Remote Sensing, vol. 47, no. 1, pp. 224-237, Jan 2009.

[25] F. Lombardini and M. Pardini, "Superresolution differential tomography: Experiments on identification of multiple scatterer in spaceborne sar data," IEEE Transactions on Geoscience and Remote Sensing, vol. 50, no. 4, pp. 1117-1129, April 2012.

[26] F. Lombardini and F. Cai, "Temporal decorrelation-robust sar tomography," IEEE Transactions on Geoscience and Remote Sensing, vol. 52, no. 9, pp. 5412-5421, Sept 2014.

[27] L. Ferro-Famil et al., "Posar: a vhr tomographic gb-sar system application to snow cover 3-d imaging at x and ku bands," in 9th European Radar Conference (EuRAD 2012), 2012, pp. 130-133. 